\title{
ArcheoSciences
}

Revue d'archéométrie

\section{Étude pétrographique et géochimique comparative du support en mortier de deux tables à marqueterie de pierres}

Comparative petrographic and geochemical study of two inlaid tables mortar supports

Suzanne Raynaud, Yannick Lacaze, Olivier Bruguier, Henri de La Boisse et René Fabre

\section{OpenEdition \\ Journals}

Édition électronique

URL : https://journals.openedition.org/archeosciences/183

DOI : 10.4000/archeosciences.183

ISBN : 978-2-7535-1595-6

ISSN : 2104-3728

Éditeur

Presses universitaires de Rennes

\section{Édition imprimée}

Date de publication : 31 décembre 2006

Pagination : 81-88

ISBN : 978-2-7535-0456-1

ISSN : 1960-1360

Référence électronique

Suzanne Raynaud, Yannick Lacaze, Olivier Bruguier, Henri de La Boisse et René Fabre, «Étude pétrographique et géochimique comparative du support en mortier de deux tables à marqueterie de pierres », ArcheoSciences [En ligne], 30 | 2006, document 7, mis en ligne le 31 décembre 2008, consulté le 31 janvier 2022. URL : http://journals.openedition.org/archeosciences/183 ; DOI : https://doi.org/ 10.4000/archeosciences.183 


\title{
Étude pétrographique et géochimique comparative du support en mortier de deux tables à marqueterie de pierres
}

\author{
Suzanne Raynaud*, Yannick Lacaze*, Olivier Bruguier**, \\ Henri DE LA BoISSE*** et René FABRE****
}

\begin{abstract}
Résumé : Les tables à marqueterie de pierre ont été créées comme catalogues pour présenter les pierres marbrières disponibles sur le marché au $\mathrm{XVII}^{\mathrm{e}}$ et XVIII ${ }^{\mathrm{e}}$ siècle. Notre étude porte sur deux tables dont le plateau est une marqueterie de pierres marbrières provenant très majoritairement de la région Languedoc-Roussillon. Dans les deux cas, la marqueterie de pierre est montée sur un support en mortier, technique de fabrication connue dans les ateliers français. Le plus célèbre de ces ateliers est l'atelier des Gobelins, ouvert entre 1645 et 1715, et dont on pense que la table de Versailles dite «table du Roi » est issue.

Les résultats des observations et des analyses des mortiers des deux tables à notre disposition (observation macroscopique, microscope polarisant, microsonde électronique, diffraction X, éléments en traces par ICP-MS) montrent qu'il s'agit de mortiers dont l'agrégat est formé de tufs volcaniques importés de la province magmatique romaine et toscane en Italie, lié par de la phillipsite.

La similitude extrême des résultats obtenus permet de conclure à la fabrication contemporaine et dans un même atelier des tables étudiées.
\end{abstract}

\begin{abstract}
Stones inlaid tables have been created to present the available marbles on the market at $17^{\text {th }}$ or $18^{\text {th }}$ centuries. Our study deals with two tables from which tops are inlaid of marbles coming in the majority of cases from Languedoc-Roussillon. The two tables show a mortar made support; a technique applied in French workshop the Atelier des Gobelins between 1645 and 1715. The table call "king table" localised to the Versailles castle was probably made in the Atelier des Gobelins.

Results of mortars analysis show that the aggregate is made of volcanic tuffs imported from Roman and Toscan magmatic province, bound with Phillipsite.

The extreme similarity of the results allows concluding that the two tables making have been contemporaneous and done in the same workshop.
\end{abstract}

Mots clés : Tables, marqueterie de pierre, mortier, tufs volcaniques, pouzzolane, éléments en traces, Italie, microsonde électronique, ICPMS.

Key words: Tables, stone inlaid, mortar, tuff, pouzzolane, traces elements, Italy, electronic microprobe, ICPMS.

\section{INTRODUCTION}

En Europe, René Fabre a déterminé la nature et la provenance des pierres marbrières et marbres de dix tables à marqueterie de pierres sur support de mortier : 5 sont localisées en France : 1 à Versailles, 1 au Louvre, 1 à la galerie de minéralogie du Museum d'Histoire Naturelle, 1 à l'Université Montpellier II, 1 au château de Flaugergues et 5 sont localisées à Rome : 2 à la

\footnotetext{
* Équipe Pierre et Monuments Géosciences Montpellier CC 060, Université Montpellier 2, 34095 Montpellier cedex 5. (suzanne.raynaud@gm.univmontp2.fr) (yopsee@free.fr)

** Service Commun ICP-MS Géosciences Montpellier CC 060, Université Montpellier 2, 34095 Montpellier cedex 5. (bruguier@gm.univ-montp2.fr)

*** Équipe Pierre et Monuments Géosciences Montpellier CC 060, Université Montpellier 2, 34095 Montpellier cedex 5. (boisse@univ-montp2.fr)

**** Architecte sculpteur, 4 av. de la Condamine, 34420 Cers. (renefabre@wanadoo.fr)
} 
villa Borghese, 2 à la Trinité des Monts et 1 au Vatican. Outre le fait que la marqueterie est réalisée sur une plaque de mortier, ce qui leur confere une originalité certaine, on trouve les mêmes variétés de pierres marbrières dans tous les cas.

D’après Palacios (1995) des tables à marqueterie de pierres et à support de mortier figurent dans l'inventaire de l'atelier des Gobelins, tandis que les tables à marqueteries de pierres sur support de schistes ou de marbres étaient réputées fabriquées en Italie. L'atelier des Gobelins est essentiellement connu du public pour ses productions en matière de tapisseries mais en 1645 sur ordre de Louis XIV, Colbert crée un atelier de fabrication de tables à marqueterie de pierres afin de servir de présentoir des différentes variétés de marbres, dont c'était la grande mode, disponibles sur le marché. Cette activité a été arrêtée en 1715 sur ordre de Louis XV. Les tables de Versailles auraient été fabriquées dans l'atelier des Gobelins.

Nous avons à notre disposition pour l'étude du mortier du support, les tables du Château de Flaugergues (TF) et la table de l'Université Montpellier II (TUMII). L'historique de ces tables n'est pas connu avec certitude. Cependant, les données en notre possession permettent d'établir les faits suivants. Henri de Colbert, propriétaire de la table de Flaugergues, possède la facture d'achat datée de 1777 indiquant que les Colbert ont acheté cette table à un abbé montpelliérain. Ceci permet de dire que cette table a été fabriquée avant cette date. L'origine de la table de l'Université Montpellier II est moins précise car elle ne figure dans aucun inventaire ancien. D'après une tradition orale interne au département de géologie, cette table provient de l'ancien évêché de Montpellier. En 1905 elle aurait été affectée, avec d'autres mobiliers anciens de l'église, à la faculté des Sciences. Depuis le déménagement de l'Université vers le campus actuel dans les années 1960 elle a été sauvegardée dans la bibliothèque du département de géologie pour sa beauté et sa richesse minéralogique.

Cette étude traite du problème de l'origine de ces deux tables. La première question est : ces deux tables, malgré leurs différences, sortent-elles du même atelier de fabrication? Etait-ce l'atelier des Gobelins? Pour apporter des éléments de réponse nous avons réalisé une description comparative pétrographique et géochimique du support de mortier de ces deux tables par une association de méthodes dont certaines rarement utilisées pour l'étude des mortiers comme l'ICPMS et la sonde électronique (Palazzo-Bertholon 2000).

\section{Caractéristiques des Marqueteries DE PIERRE}

Les pierres des marqueteries de toutes ces tables proviennent du bassin méditerranéen : depuis les pierres marbrières du Languedoc et des Pyrénées, les plus nombreuses, jusqu'aux porphyres rouges d'Egypte, la brocatelle d'Espagne, l'onyx d'Algérie, les marbres et porphyres verts de Grèce, l'Africano de Turquie, les jaspes de Sicile, les lapis lazuli d'Afghanistan, les marbres d'Italie. Il figure en outre sur la table du château de Flaugergues une pierre bleue inventée, semble-t-il, à l'époque de Côme $1^{\text {er }}$ de Médicis, qui serait composée de poudre de lapis lazuli, de colle et de marbre.

Bien que regroupant des marbres de mêmes origines, les deux tables diffèrent par leurs dimensions (Tableau 1), la disposition des carreaux et les choix d'associations de couleur (Fig. 1). La table de Flaugergues, plus grande, est faite de carreaux, disposés en quinconce, délimités par de fines baguettes de marbre noir, dit "de Belgique ", dont est également composée la bordure extérieure. La table de l'UMII, plus petite, présente des carreaux parallèles aux bordures et jointifs entre eux. Sa bordure extérieure comprend une fine baguette de noir de Belgique (ou de colonnata) vers l'intérieur et une bordure extérieure en jaune de Sienne. Dans les deux cas, l'habillage latéral vertical est de la même pierre que le bandeau extérieur et cache le support en mortier visible sous la table. Nous ne nous sommes pas intéressés à l'étude des cadres et des pieds en bois des tables qui ne sont probablement pas d'origine.

\begin{tabular}{|l|l|l|}
\hline Mesures en cm & $\begin{array}{l}\text { Table de } \\
\text { Flaugergues }\end{array}$ & $\begin{array}{l}\text { Table de l'UM } \\
\text { II }\end{array}$ \\
\hline Épaisseur de la pierre & 0,4 & 0,4 \\
\hline Largeur totale & 88,5 & 57,3 \\
\hline Largeur bandeau extérieur & 6,2 & 0,7 et 3,5 \\
\hline Longueur totale & 175,8 & 138,2 \\
\hline Nombre de carreaux & $\begin{array}{l}34 \text { demis, } \\
4 \text { quarts, } \\
138 \text { entiers }\end{array}$ & 180 \\
\hline Taille carreaux & 7,5 à 7,2 x 7,2 & $\begin{array}{l}6,3 \text { à } 6,5 \text { x } 6,3 \\
\text { à } 6,5\end{array}$ \\
\hline $\begin{array}{l}\text { Largeur baguette noire inter- } \\
\text { carreaux }\end{array}$ & 1,1 à 1,3 & \\
\hline
\end{tabular}

Tableau 1 : Les dimensions des tables. Table 1: Table dimensions.

\section{MÉTHOdes D'ÉTUdE DU MORTIER DU SUPPORT}

Les échantillons, d'environ $10 \mathrm{~g}$, ont été prélevés sous la table par un carottage discret. Nous avons utilisé des méthodes permettant une caractérisation pétrographique, texturale et géochimique. L'observation macroscopique a permis de décrire la nature et les proportions des différents constituants du mortier. Sur des lames minces le microscope optique 


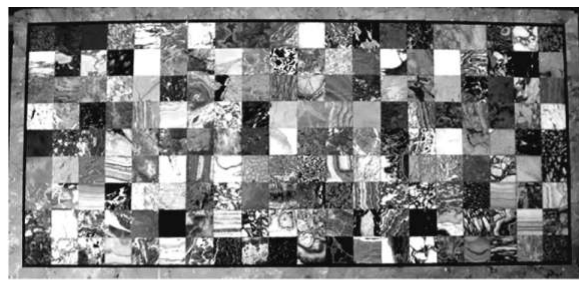

T UMII

TF

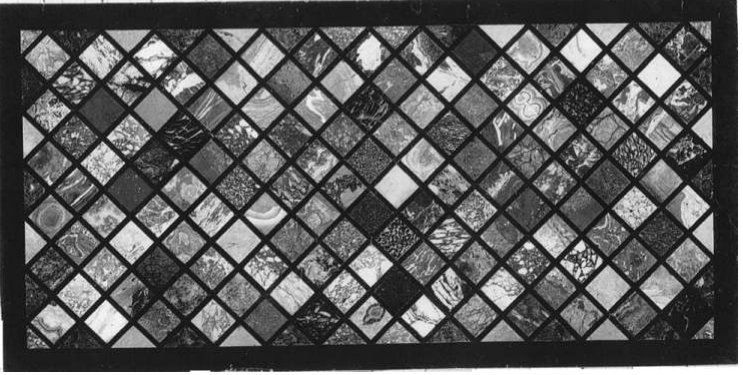

Figure 1: Photo des plateaux des deux tables. T F : table de Flaugergues, T UMII : table de l'Université Montpellier II.

Figure 1: Tops of the two tables. TF: Flangergues table, TUMII: Montpellier II University table.

nous a permis de décrire la proportion de granulat/liant, les principaux minéraux présents dans le granulat (type, taille, forme, quantité) ainsi que la nature du liant.

Pour préciser la composition minéralogique exacte de certains minéraux nous avons utilisé une microsonde électronique à dispersion de longueur d'ondes CAMECA SX100. La mesure du rayonnement $\mathrm{X}$ induit par bombardement électronique permet l'analyse chimique qualitative et quantitative dans un très petit volume de matière, de l'ordre du micron cube.

Pour les analyses par diffraction X et ICP-MS les échantillons ont été broyés en poudre fine dans un mortier en agate, puis tamisés pour ne garder que la fraction inférieure à $80 \mu \mathrm{m}$, afin de concentrer le liant par rapport à l'agrégat. La diffraction $\mathrm{X}$ a permis de préciser la nature des composants des liants.

La technique d'analyse des éléments en trace nous a semblé utile pour différencier les liants des mortiers d'apparence semblable. Elle a été réalisée par Spectrométrie de Masse à Plasma Inductif (ICP-MS) à l'aide d'un appareil de type VG PQ II Turbo muni d'une Option $S$ permettant un gain de sensibilité. Après pesée, les échantillons ont été dissous par voie acide dans un mélange $\mathrm{HF}-\mathrm{HClO}_{4}$ (3:1). La dissolution a été réalisée pendant 48 heures sur plaque chauffante dans des béchers en téflon. L'ajout d'acide perchlorique permet de porter le mélange acide à un degré d'oxydation maximum, tout en élevant de façon significative sa température d'ébullition. Enfin, il permet également d'éviter la formation de fluorures insolubles (en particulier fluorures de Ca) susceptibles de piéger les éléments en traces. Ceci conduit a une solution limpide qui est mise à évaporer à environ $150{ }^{\circ} \mathrm{C}$ sur plaque chauffante. Une deuxième étape de dissolution est alors réalisée avec des quantités d'acide réduites de moitié. Après cette deuxième phase de dissolution, l'échantillon est alors soumis à trois étapes d'évaporation successives avec ajout d' $\mathrm{HClO}_{4}$ dans des quantités décroissantes. Ceci permet d'éliminer toutes traces de fluor résiduel issu de l'ajout d'acide fluorhydrique lors des deux étapes de dissolution. La mesure des concentrations des éléments en trace suit la méthode décrite par Ionov et al. (1992) et seuls quelques points sont rappelés brièvement ci-dessous. Après dilution des échantillons, une quantité connue de standards interne (In et $\mathrm{Bi}$ ) est rajoutée qui permet de contrôler la dérive de la machine ainsi que les effets de matrice. L'analyse par ICP-MS se fait en mode saut de pic (3 points par pic). Les concentrations sont obtenues par calibration externe grâce à l'analyse de solutions multi-élémentaires contenant les éléments à analyser et qui sont préparées à partir de solutions mono-élémentaire. La calibration du Nb et du Ta a été réalisée par calibration auxilliaire en utilisant le $\mathrm{Zr}$ et le $\mathrm{Hf}$ respectivement suivant la méthode décrite par Jochum et al. (1990). La précision des mesures est généralement meilleure que $5 \%$ (1б RSD) et la justesse, contrôlée par la mesure de standards de roche naturelle (BEN, UBN et CAL-S), est meilleure que $10 \%$.

\section{Résultats}

\subsection{Description macroscopique du mortier des deux tables}

L'observation montre un liant gris clair pour environ $30 \%$ de la surface d'observation. Les éléments du granulat sont anguleux, hétérométriques et polygéniques. On observe des éléments noirs de taille inférieure à $2 \mathrm{~mm}$, des éléments blancs jusqu'à $5 \mathrm{~mm}$, des éléments translucides et des éléments marron à beige de la même classe de taille. Comme le montre la figure 2 l'aspect macroscopique est le même pour les deux mortiers dont la surface est rugueuse.

\subsection{Minéralogie}

Comme observé macroscopiquement, l'étude microscopique confirme la similitude entre les mortiers des deux tables. Elle fournit en outre les informations suivantes :

ArCheoSciences, revue d'archéométrie, 30, 2006, p. 81-88 

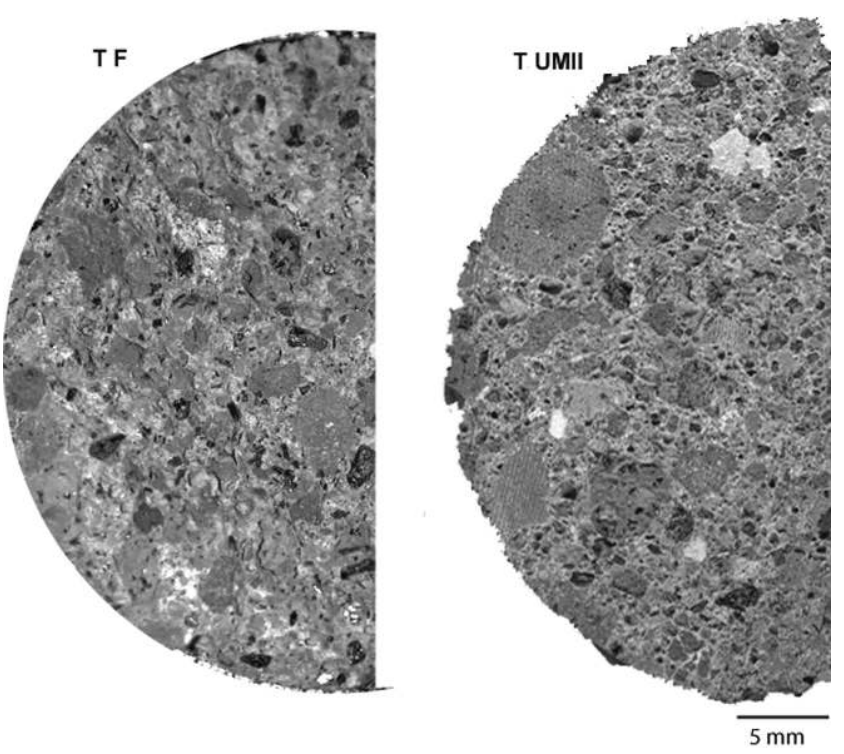

Figure 2: Photographie macroscopique des supports en mortier. T F : table de Flaugergues, T UMII : table de l'Université Montpellier II.

Figure 2: Macroscopic photography of mortar supports. TF: Flangergues table, TUMII: Montpellier II University table.

- Le granulat (Fig. 3) est formé de fragments lithiques de tufs volcaniques (cinérite plus ou moins ponceuse et plus ou moins altérée) contenant des pyroxènes, augites ægyriniques souvent zonées (Fig. 4), du verre volcanique, plus rarement du plagioclase, de l'olivine, et des amphiboles brunes. Les minéraux précédents, surtout lorsqu'ils sont de grande taille, peuvent être isolés dans le liant. Ils sont accompagnés de rares débris de calcaire micritique et de calcite.

- Le liant est formé de cristaux aciculaires translucides, à extinction droite et biréfringence faible, souvent regroupés en éventails. Ces caractéristiques minéralogiques évoquent une nature zéolitique (Fig. 5). Les résultats de diffraction X montrent pour les deux tables une grande complexité dans la composition du liant gris : de la phillipsite calcique (variété de zéolite) et de la calcite ainsi que de nombreux constituants plus difficilement identifiables. La seule différence observable entre la composition des deux tables est la présence d'un peu de dolomie ferrifere dans celle de Flaugergues. À ce stade de l'étude, une très forte ressemblance entre ces deux mortiers est évidente.

\section{3. Étude géochimique du mortier}

\subsubsection{Microsonde électronique}

Les résultats d'analyse obtenus grâce à la microsonde électronique permettent de mieux cerner l'origine du matériel

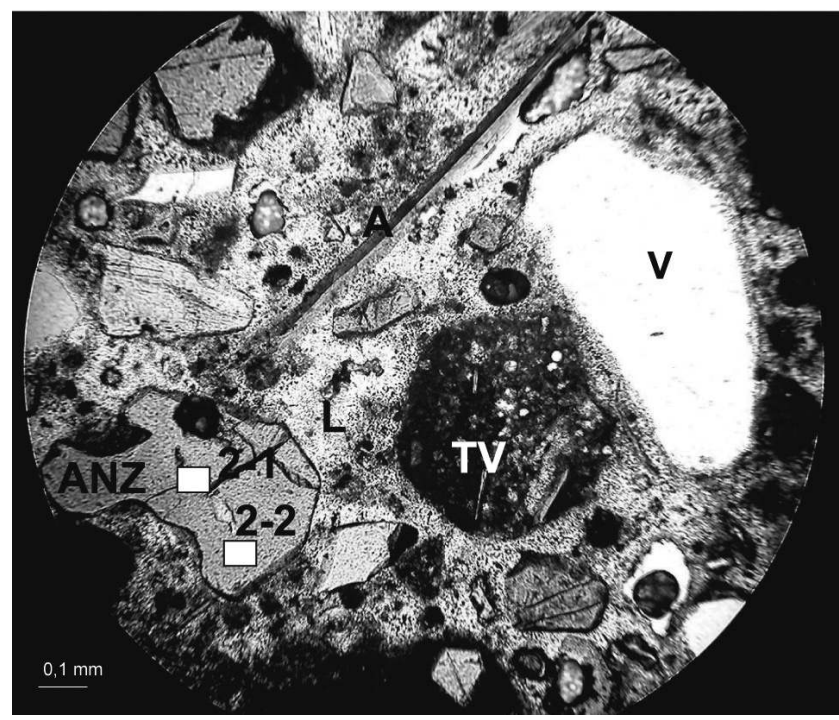

Figure 3 : Vue du mortier au microscope optique en lumière naturelle. $\mathrm{V}$ : fragment de verre volcanique, AZN : augite non zonée, $\square 1$ et 2 : localisation des analyses à la microsonde électronique, $A$ : amphibole, TV : morceau de tuf volcanique, $\mathrm{L}$ : liant.

Figure 3: Microscopic natural light view of the mortar. V: volcanic glass piece, $A Z N$ : un-zoned augite, $\square 1$ and 2: electronic microprobe analysis localization, $A$ : amphibole, TV: piece of tuff, $L$ : binder.

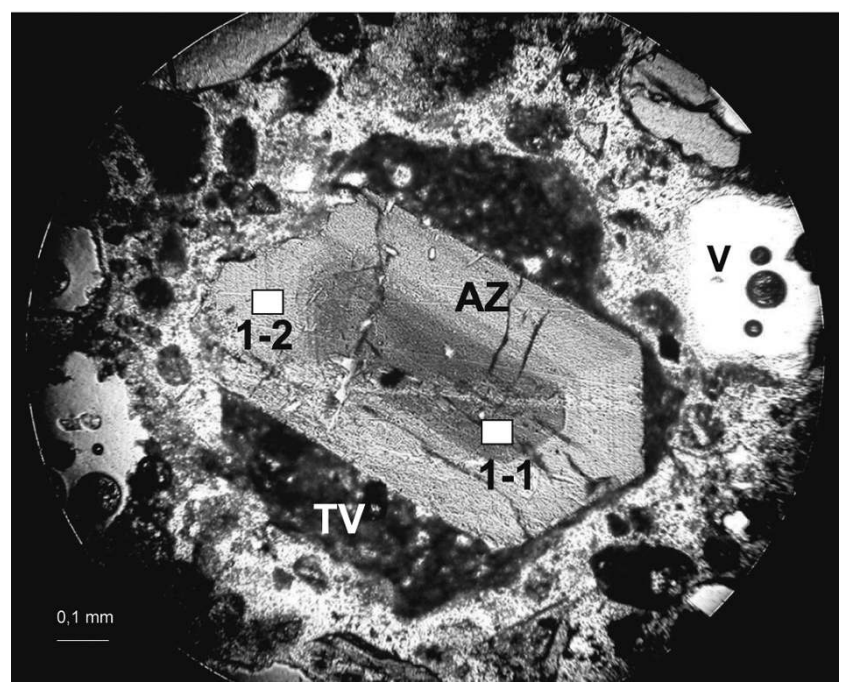

Figure 4: Vue d'une augite zonée au microscope optique en lumière naturelle. $\mathrm{AZ}$ : augite zonée au cœur d'un fragment de TV : morceau de tuf volcanique, $\mathrm{V}$ : verre volcanique, $\square 1$ et 2 : localisation des analyses à la microsonde électronique.

Figure 4: Optical natural light microscope view of a zoned augite. AZ: zoned augite in a fragment of TV: piece of tuff, $V$ : volcanic glass. $\square 1$ and 2: electronic microprobe analysis localization.

utilisé lors de la fabrication de ce mortier. Des clinopyroxènes et des zéolites observés respectivement dans le granulat et le liant du mortier de la table TUMII ont été analysés. Dans 


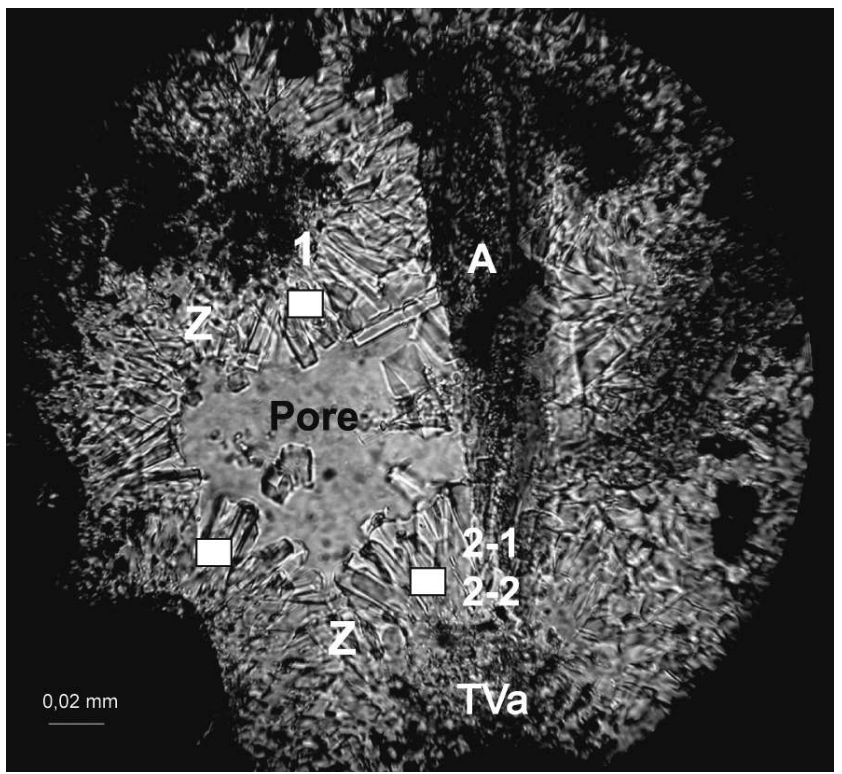

Figure 5 : Vue du liant au microscope optique en lumière naturelle. $\mathrm{Z}$ : zéolithes en couronnes autour de débris de pouzzolane altérée, TVa : tuf volcanique altéré, A : amphibole $\square 1$ : localisation des analyses à la microsonde électronique.

Figure 5: Optical natural light view of binder. Z: crowned zeolites around altered tuff fragments. Tva: altered tuff, A: amphibole, $\square$ 1: electronic microprobe analysis localization.

le granulat les analyses ont porté sur un cristal de pyroxène zoné (Fig. 4) et un cristal non zoné (Fig. 3). Dans le liant, les analyses ont été focalisées sur deux cristaux de zéolite (Fig. 5). Les résultats de ces analyses sont reportés dans le Tableau 2.

Les caractéristiques chimiques des clinopyroxènes varient suivant la composition de la lave à partir de laquelle ils ont cristallisé (Kushiro, 1960). Cette propriété a été utilisée pour retrouver les affinités magmatiques des roches et les différents contextes tectoniques dans lesquels elles se sont formées (Nisbet and Pearce, 1977; Leterrier et al., 1982). Les deux clinopyroxènes ont des compositions d'augite. Reportés dans un diagramme de discrimination (Fig. 7) ils sont situés près du champ des pyroxènes des basaltes alcalins défini par Leterrier et al. (1982).

La zéolite présente dans le liant des deux tables TUMII et TF est de la phillipsite. Elle se localise près du champ des phillipsites des tuffs volcaniques : Tuff Jaune Napolitain (TYN) (De Gennaro and Langella, 1996) (Fig. 6). On remarque toutefois que les phillipsites analysées sont nettement plus calciques (Tableau 2). Cette zéolite est connue comme produit d'altération des verres volcaniques (De Gennaro et al., 2000), ou des argiles et minéraux argileux (Ramirez et al., 2005). Elle existe aussi sous forme authigène,

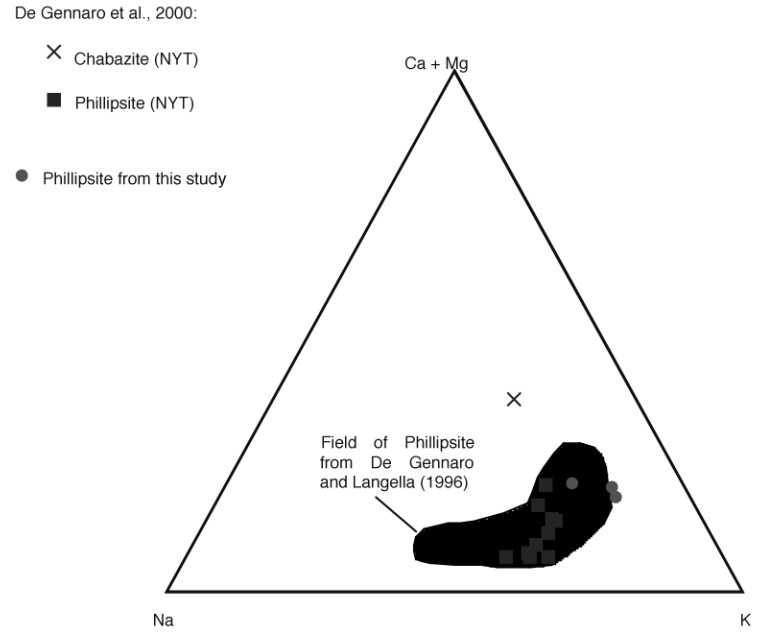

Figure 6 : Diagramme de discrimination des pyroxènes. Figure 6: Pyroxenes discrimination diagram.

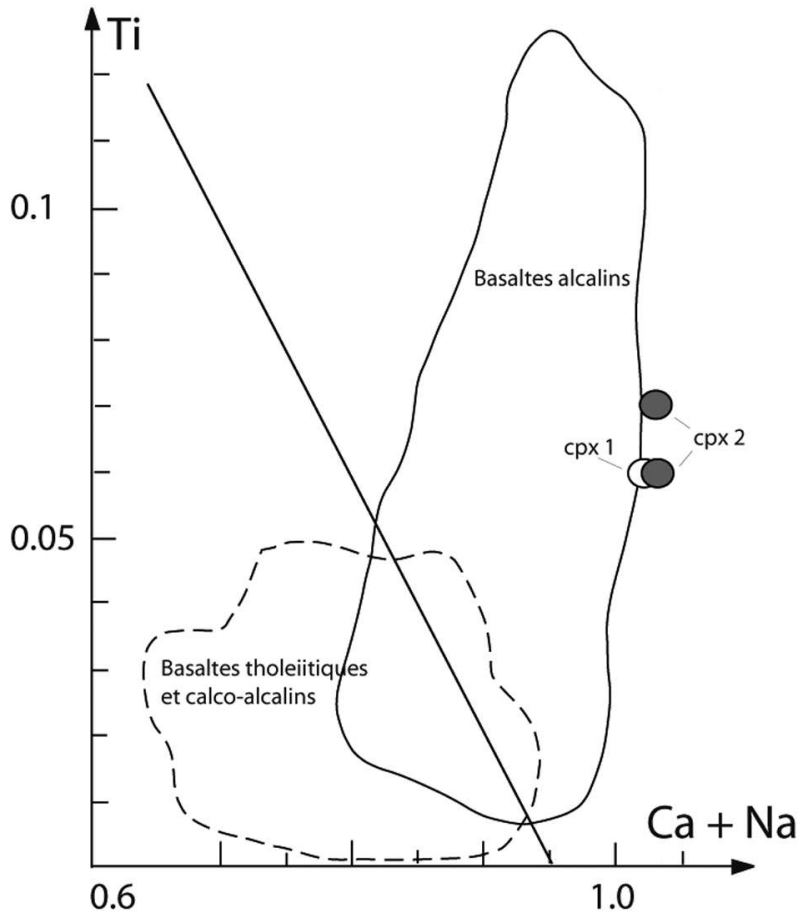

Figure 7 : Localisation des pyroxènes par rapport aux domaines des basaltes.

Figure 7: Pyroxenes localisation versus basalts domains.

formée à faible température dans les nodules de manganèse (Lee and Lee, 1998), dans les sédiments calcaires de mer profondes (Young, 1939) et sur les parois des bains romains où les sources chaudes ont une température maximum de $70^{\circ}$ (Daubrée, 1879). 
Suzanne RaynaUd, Yannick Lacaze, Olivier BrUGUIER, Henri de LA BoIsSe, René Fabre

\begin{tabular}{|l|r|r|r|r|r|r|r|}
\hline Sample & $\begin{array}{r}\text { Pyroxene 1-1 } \\
\text { zoné }\end{array}$ & $\begin{array}{r}\text { Pyroxene 1-2 } \\
\text { zoné }\end{array}$ & $\begin{array}{r}\text { Pyroxene 2-1 } \\
\text { n. zoné }\end{array}$ & $\begin{array}{r}\text { Pyroxene 2-2 } \\
\text { n. zoné }\end{array}$ & Phillipsite 1 & Phillipsite 2-1 & Phillipsite 2-2 \\
\hline $\mathrm{SiO}_{2}$ & 40,4 & 41,0 & 38,6 & 40,7 & 48,4 & 46,9 & 53,4 \\
\hline $\mathrm{Al}_{2} \mathrm{O}_{3}$ & 10,21 & 10,39 & 11,88 & 10,68 & 24,94 & 22,55 & 23,68 \\
\hline $\mathrm{FeO}$ & 15,33 & 12,66 & 13,62 & 12,45 & 0,15 & 0,68 & 0,33 \\
\hline $\mathrm{MgO}$ & 6,61 & 8,16 & 7,40 & 8,20 & 0,01 & 0,34 & 0,00 \\
\hline $\mathrm{CaO}$ & 23,21 & 23,53 & 23,43 & 23,73 & 3,72 & 4,55 & 3,07 \\
\hline $\mathrm{Na}_{2} \mathrm{O}$ & 0,45 & 0,30 & 0,26 & 0,25 & 1,30 & 2,37 & 1,28 \\
\hline $\mathrm{K}_{2} \mathrm{O}$ & 0,00 & 0,00 & 0,01 & 0,00 & 9,87 & 11,77 & 9,59 \\
\hline $\mathrm{Cr}_{2} \mathrm{O}_{3}$ & 0,01 & 0,00 & 0,03 & 0,00 & 0,00 & 0,02 & 0,01 \\
\hline $\mathrm{MnO}$ & 0,54 & 0,27 & 0,23 & 0,23 & 0,00 & 0,01 & 0,04 \\
\hline $\mathrm{TiO}_{2}$ & 1,83 & 1,91 & 2,23 & 1,91 & 0,07 & 0,08 & 0,03 \\
\hline $\mathrm{Total}$ & 98,56 & 98,25 & 97,73 & 98,16 & 88,45 & 89,25 & 91,41 \\
\hline & & & & & & & 10,04 \\
\hline $\mathrm{Si}$ & 1,62 & 1,62 & 1,55 & 1,61 & 10,14 & $10,70,70$ \\
\hline $\mathrm{Al}$ & 0,48 & 0,48 & 0,56 & 0,50 & 6,16 & 5,69 & 5,59 \\
\hline $\mathrm{Fe}$ & 0,51 & 0,42 & 0,46 & 0,41 & 0,03 & 0,12 & 0,06 \\
\hline $\mathrm{Mg}$ & 0,39 & 0,48 & 0,44 & 0,48 & 0,00 & 0,11 & 0,00 \\
\hline $\mathrm{Ca}$ & 0,99 & 1,00 & 1,01 & 1,01 & 0,84 & 1,04 & 0,66 \\
\hline $\mathrm{Na}$ & 0,03 & 0,02 & 0,02 & 0,02 & 0,53 & 0,98 & 0,50 \\
\hline $\mathrm{K}$ & 0,00 & 0,00 & 0,00 & 0,00 & 2,64 & 3,21 & 2,45 \\
\hline $\mathrm{Ti}$ & 0,06 & 0,06 & 0,07 & 0,06 & 0,01 & 0,01 & 0,00 \\
\hline $\mathrm{Cr}$ & 0,00 & 0,00 & 0,00 & 0,00 & 0,00 & 0,00 & 0,00 \\
\hline $\mathrm{Mn}$ & 0,02 & 0,01 & 0,01 & 0,01 & 0,00 & 0,00 & 0,01 \\
\hline
\end{tabular}

Tableau 2 : Résultats d'analyses à la microsonde électronique (wt\%) et composition atomique des pyroxènes et zéolites identifiés dans le ciment de la table de l'UMII. Les points d'analyse sont localisés figure 3 pour l'augite non zonée, figure 4 pour l'augite zonée et figure 5 pour la phillipsite. Les formules des pyroxènes et de la phillipsite sont calculées respectivement sur 6 et 32 atomes d'O. Table 2: Electron microprobe analyses (wt\%) results and atomic contents of pyroxenes and zeolites identified in the cement of the inlaid table of UMII. Pyroxene and phillipsite formula is calculated on 6 and $32 O$ atoms respectively. The analysed points are localised figure 3 for the un-zoned augite and figure 5 for the phillipsite.

\subsubsection{ICP-MS}

Les analyses des éléments en trace apportent trois résultats majeurs.

(1) Une superposition parfaite des spectres des mortiers des deux tables, normalisés par rapport à un réservoir de référence (Manteau Primitif) (Fig. 8). Ils sont marqués par un enrichissement qui diminue progressivement depuis le Th jusqu'au $\mathrm{Lu}$, par de fortes anomalies négatives en $\mathrm{Nb}$, $\mathrm{Ta}$ et par des anomalies positives en $\mathrm{Pb}$ et $\mathrm{Sr}$. Les deux mortiers sont caractérisés par un enrichissement en éléments incompatibles lithophiles $(\mathrm{Ba}, \mathrm{Th}, \mathrm{U})$ ainsi qu'en Terres Rares légères $(\mathrm{La}, \mathrm{Ce})$.

(2) Une remarquable similitude entre les spectres des mortiers des deux tables d'une part, et ceux des roches volcaniques des Provinces Romaine et Toscane en Italie d'autre part (Gasperini et al., 2002), à l'exception d'une légère anomalie positive en Sr (Fig. 8 et Tableau 3). Les teneurs d'élément en traces (Tableau 3) et les spectres (Fig. 8) contrastent par contre nettement avec ceux obtenus sur des échantillons de chaux pure actuelle aérienne (A, type CAEB, NF P15510) et

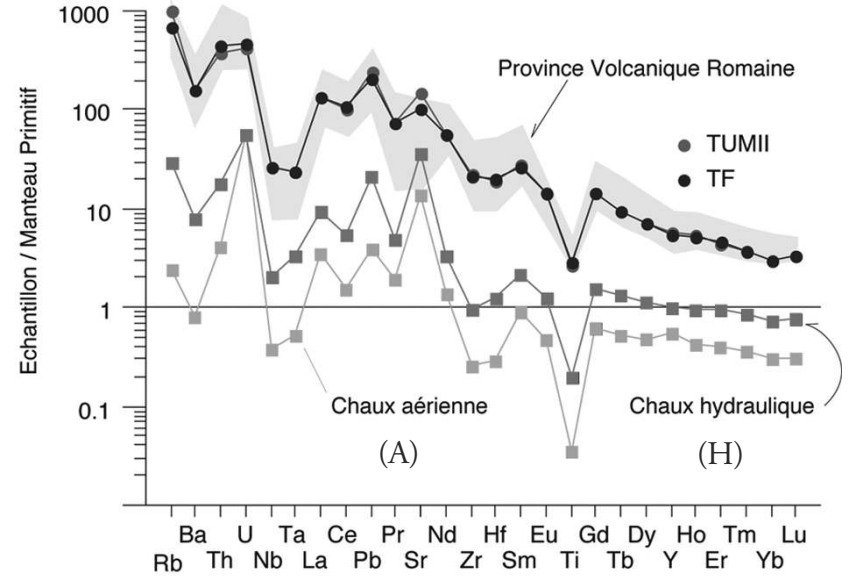

Figure 8 : Spectres des éléments en trace des tables TF et TUMII; comparaison avec la chaux hydraulique $(\mathrm{H})$ et aérienne $(\mathrm{A})$. Figure 8: Trace element patterns of tables TF and TUMII and comparison with an hydraulic $(H)$ and normal lime $(A)$. 
hydraulique (H, type NHL 3.5, NF P15311). Ces derniers présentent des teneurs en éléments en traces significativement moins élevées et des spectres moins fractionnés.

(3) Les valeurs $\mathrm{Nb} / \mathrm{Ta}$ des mortiers des tables (Tableau 3) sont comprises entre 19 et 20 ce qui est proche des valeurs de basaltes alcalins de type OIB (Kalfoun, 2002). Les rapports $\mathrm{Nb} / \mathrm{Ta}$ de la chaux aérienne (A) et de la chaux hydraulique $(\mathrm{H})$ sont compris entre 11 et 13 , voisins de ceux de la croûte continentale (11 \pm 2 , Barth et al., 2000) ce qui est en bon accord avec la fabrication de ces chaux à partir de matériaux naturels d'origine principalement crustale (carbonate plus ou moins argileux).

\begin{tabular}{|c|c|c|c|c|}
\hline Sample Name & $\mathrm{H}$ & A & TUMII & TF1 \\
\hline $\mathrm{Rb}$ & 18,45 & 1,52 & 628,61 & 431,03 \\
\hline $\mathrm{Sr}$ & 771,99 & 286,92 & 3195,20 & 2140,60 \\
\hline Y & 4,62 & 2,58 & 26,39 & 24,46 \\
\hline $\mathrm{Zr}$ & 10,58 & 2,93 & 245,88 & 241,59 \\
\hline $\mathrm{Nb}$ & 1,45 & 0,27 & 19,06 & 18,69 \\
\hline Cs & 1,50 & 0,13 & 34,77 & 37,76 \\
\hline $\mathrm{Ba}$ & 57,17 & 5,65 & 1117,20 & 1127,10 \\
\hline $\mathrm{La}$ & 6,53 & 2,40 & 90,03 & 91,15 \\
\hline $\mathrm{Ce}$ & 9,65 & 2,73 & 180,39 & 192,10 \\
\hline $\operatorname{Pr}$ & 1,36 & 0,54 & 20,50 & 20,42 \\
\hline $\mathrm{Nd}$ & 4,61 & 1,86 & 76,50 & 76,19 \\
\hline Sm & 0,98 & 0,41 & 12,14 & 11,98 \\
\hline $\mathrm{Eu}$ & 0,21 & 0,08 & 2,46 & 2,48 \\
\hline $\mathrm{Gd}$ & 0,95 & 0,38 & 8,47 & 8,50 \\
\hline $\mathrm{Tb}$ & 0,14 & 0,06 & 1,02 & 1,02 \\
\hline Dy & 0,84 & 0,35 & 5,14 & 5,17 \\
\hline Ho & 0,16 & 0,07 & 0,87 & 0,85 \\
\hline $\mathrm{Er}$ & 0,45 & 0,19 & 2,14 & 2,17 \\
\hline $\mathrm{Tm}$ & 0,06 & 0,03 & 0,27 & 0,28 \\
\hline $\mathrm{Yb}$ & 0,35 & 0,16 & 1,50 & 1,52 \\
\hline $\mathrm{Lu}$ & 0,06 & 0,02 & 0,25 & 0,25 \\
\hline $\mathrm{Hf}$ & 0,39 & 0,09 & 5,82 & 6,31 \\
\hline $\mathrm{Ta}$ & 0,14 & 0,02 & 0,97 & 0,98 \\
\hline $\mathrm{Pb}$ & 3,91 & 0,74 & 43,95 & 37,52 \\
\hline Th & 1,56 & 0,35 & 31,64 & 36,63 \\
\hline U & 1,21 & 1,18 & 8,90 & 9,68 \\
\hline $\mathrm{Ti}$ & 255,85 & 45,74 & 3415,80 & 3672,60 \\
\hline $\mathrm{Nb} / \mathrm{Ta}$ & 10,7 & 12,6 & 19,7 & 19,1 \\
\hline
\end{tabular}

Tableau 3 : Données sur les éléments en trace dans le mortier des tables à marqueterie (TUMII, TF) mesurées par ICP-MS. Pour comparaison figurent la chaux hydraulique et la chaux aérienne actuelles ( $\mathrm{H}$ : chaux hydraulique; A: chaux normale).

Table 3: Traces elements ICP-MS data of mortar from the inlaid tables (TUMII, TF1). For comparison present day limes have also been studied (H: hydraulic lime; A: normal lime).
Les éléments incompatibles comme le $\mathrm{Nb}$ et le $\mathrm{Ta}$ fractionnent difficilement entre eux au cours des processus géologiques naturels. Ils peuvent donc être considérés comme des marqueurs importants de la nature des matériaux initiaux utilisés pour la confection des mortiers et confirment pour les tables étudiées la provenance volcanique de ces matériaux.

\section{Discussion ET CONCLUSION}

Les résultats obtenus sur le mortier des deux tables montrent l'extrême similitude de leur nature et de la provenance de leurs composants.

L'agrégat des mortiers est composé de tufs volcaniques altérés de type basaltes des îles océaniques (OIB), comme cela est démontré par les analyses des pyroxènes à la microsonde électronique et les valeurs des rapports $\mathrm{Nb} / \mathrm{Ta}$ obtenues à l'ICP-MS. La composition générale de ces roches permet de les rattacher au volcanisme italien de la région de Toscane et de Rome (Volmer, 1976; Hamelin et al., 1979), et donc à l'exploitation classique de ces tufs comme pouzzolanes pour les mortiers spéciaux depuis l'époque romaine.

Le liant est essentiellement formé de phillipsite. Cette zéolite se présente en éventail autour des éléments de l'agrégat sous forme géodique, et s'est donc formée in situ, par réaction entre l'agrégat et le liant lors de la prise du mortier. Sa provenance est essentiellement liée aux argiles d'altération du tuf. La phillipsite a hérité du cortège des éléments en traces de ces argiles, dont le spectre est très semblable à celui, là encore, des roches volcaniques des provinces Romaine et Toscane d'Italie. Ces zéolites sont beaucoup plus calciques que les phillipsites naturelles, ce qui indiquerait la présence de chaux dans le mélange initial.

Il n'y a pas trace d'autres constituants. Il semble donc que le mortier analysé soit le résultat du mélange d'un agrégat pulvérulent de pouzzolanes italiennes, de chaux et d'eau.

On peut donc en conclure que les fabricants de ce mortier n'ont pas hésité à utiliser des produits d'excellente qualité, quitte à importer la pouzzolane à partir de ses meilleurs sites d'extraction si les tables ont été créées en France.

Le résultat majeur de cette étude est la ressemblance extrême des caractéristiques macroscopiques, microscopiques et minéralogiques des mortiers des deux tables. Cette ressemblance est totalement corroborée par les spectres des éléments en traces, indissociables à l'intérieur de la marge d'erreur analytique. La seule explication que l'on puisse apporter est l'affirmation que la fabrication de ces tables a été contemporaine, effectuée à partir des mêmes matériaux et avec les mêmes dosages. On doit donc en conclure que les 
tables à marqueterie de pierre de Flaugergues et de l'Université Montpellier II sont issues du même atelier.

\section{Bibliographie}

Barth, M. G., McDonough, W. F. et Rudnick, R. L., 2000. Tracking the budget of $\mathrm{Nb}$ and $\mathrm{Ta}$ in the continental crust, Chemical Geology, v. 165, p. 197-213.

Daubrée, A., 1879. Études synthétiques de géologie expérimentale, Paris.

De'Gennaro, M., and Langella, A., 1996. Italian zeolitized rocks of technological interest, Mineralium Deposita, v. 31, p. $452-472$.

De'Gennaro, M., Cappelletti, P., Langella, A., Perrotta, A. et Scarpati, C., 2000. Genesis of zeolites in the Neapolitan Yellow Tuff: geological, volcanological and mineralogical evidence, Contibutions to Mineralogy and Petrology, v. 139, p. 17-35.

Gasperini, D., Blichert-Toft, J., Bosch, D., Del Moro, A., Macera, P. et Albarede, F., 2002. Upwelling of deep mantle material through a plate window: evidence from the geochemistry of Italian basaltic volcanics, Journal of Geophysical Research, v. 107, p. 2367-2385.

Hamelin, B., Lambret, B., Joron, J. L., Treuil, M. et Allegre, C. J., 1979. Geochemistry of basalts from the Tyrrhenian Sea, Nature, v. 278, p. 832-834.

Ionov, D., Savoyant, L. et Dupuy, C., 1992. Application of the ICP-MS technique to trace element analysis of peridotite and their minerals, Geostandards Newsletter, v. 16, p. 311-315.

Jochum, K. P., Seufert, H. M. et Thirlwall, M. F., 1990. Highsensitivity $\mathrm{Nb}$ analysis by spark-source mass spectrometry (SSMS) and calibration of XRF $\mathrm{Nb}$ and $\mathrm{Zr}$, Chemical Geology, v. 81 , p. $1-16$.
Kalfoun, F., 2002. Géochimie du Niobium et du tantale : distribution et fractionnement de ces deux éléments dans les différents réservoirs terrestres. thèse de doctorat, Université de Montpellier II, $275 \mathrm{p}$.

Kushiro, I., 1960. Si-Al relation in clinopyroxenes from igneous rocks, American Journal of Sciences, v. 258, p. 548-554.

LeE, C. H. et LeE, S. R., 1998. Authigenic phillipsite in deepsea manganese nodules from the Clarion-Clipperton area, NE equatorial Pacific, Marine Geology, v. 148, p. 125-133.

Leterrier, J., Maury, R. C., Thonon, P., Girard, D. et Marchal, M., 1982. Clinopyroxene composition as a method of identification of the magmatic affinities of paleo-volcanic series. Earth and Planetary Science Letters, v. 59, p. 139-154.

Nisbett, E. G. et Pearce, J. A., 1977. Clinopyroxene composition in mafic lavas from different tectonic settings, Contributions to Mineralogy and Petrology, v. 63, p. 149-160.

Palacios, A. G. 1995. Y fiorentini del re sole il gusto dei principi arte di corte del 17 et del 18eme seculo, Tome 1, Milan, Ed. Arte di Corte Longanesi, p. 1960.

Palozzo, Bertholon 2000. Archéométrie et Archéologie des mortiers et des enduits peints médiévaux. Étude critique de la bibliographie, Archéologie Médiévale, tome 29, CNRS éditions, p. 191-216.

Ramirez, S., Vieillard, P., Bouchet, A., Cassagnabere, A., Meunier, A et JacQuot, E., 2005. Alteration of the CallovoOxfordian clay from Meuse-Haute Marne underground laboratory (France) by alkaline solution, I. A XRD and CEC study, Applied Geochemistry, v. 20, p. 89-99.

Vollmer, R., 1976. Rb-Sr and U-Th-Pb systematics of alkaline rocks: the alkaline rocks from Italy, Geochimica et Cosmochimica Acta, v. 40, p. 283-295.

Young, J. A., 1939. Minerals from deep sea cores and surface deposits of Bermudian calcareous sediments, American Journal of Sciences, v. 237, p. 798. 